\title{
Frontal Asymmetry Changes Following Passive Hypo-Hydration
}

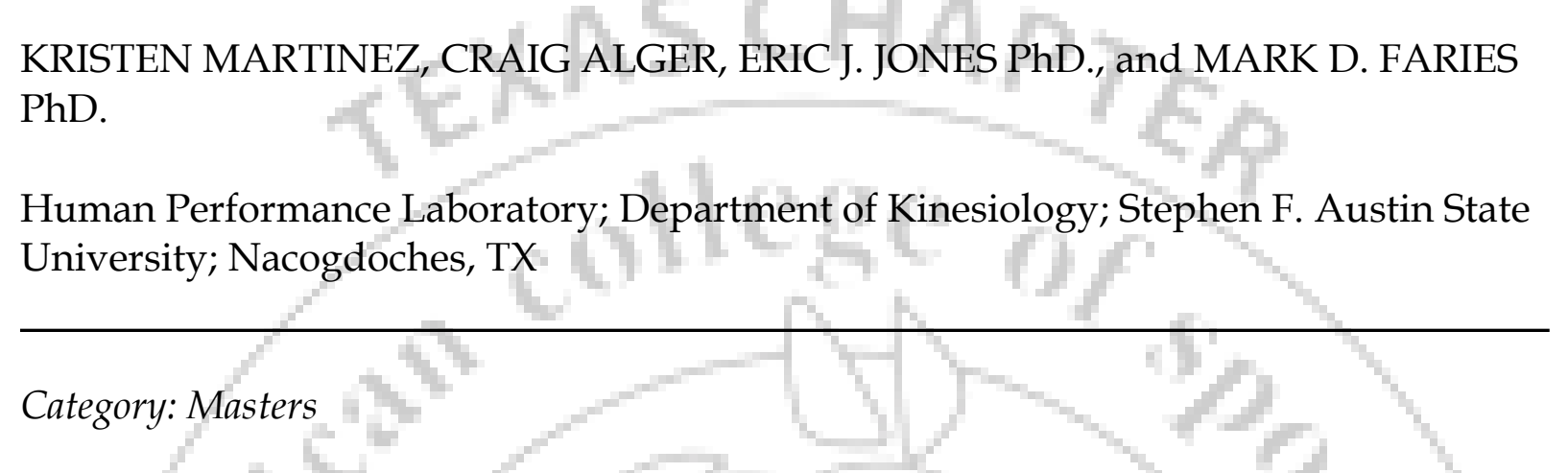

INTRODUCTION: Universal fluid consumption prescriptions among both athletic and non-athletic populations continue to elicit both hypo- and hyper-hydration (hypo-natremia). Thus, the need to find novel, identifiable traits within individuals who may be at higher risk to experience hypo-hydration, as well as hypo-natremia, is warranted. Of interest here, behavior is encapsulated by two motivational systems, referred to as approach/reward (R) or avoidance/non-reward (NR), respectively, in response to a behavioral stimulus. Past literature has revealed individual differences in consumptive traits of various types of foods between these motivational systems. Therefore, the purpose of this study was to evaluate changes in R/NR via frontal asymmetry (FA) in response to water exposure during eu-hydrated and hypo-hydrated conditions. METHODS: Participants included 11 active college-aged males. Electroencephalogram (EEG) assessed motivational orientations to water exposure in both eu-hydrated and hypo-hydrated states. A 5-minute nature video was used to stabilize mood, followed by 2 minutes of resting EEG recordings. Then, FA was assessed with the presentation of a glass of ice water, with instructions to observe, but not consume for 3 minutes. FA was re-assessed in the same manner following a dehydration trial ( $\sim 90$ minutes). Dehydration trials were performed in a controlled hot water bath at a mean of $39^{\circ} \mathrm{C}$. Participants remained in the bath until $2 \%$ body mass loss, with no fluids consumed during the trials. Urine voids were collected before and after trials to assess specific gravity and color. Body core temperature was monitored throughout the protocol, with $>38.7^{\circ} \mathrm{C}$ set as criteria for removal of the subject. Participants reported perceptual affective measures using Feeling Scale (FS) and Felt Arousal Scale (FAS) throughout the session to account for any unusual affective states. RESULTS: EEG recordings were applied to the Frontal Asymmetry Index (FAI) (log right alpha power minus log left alpha power). Higher scores on the FAI indicate higher relative left frontal lobe activity, corresponding with an affinity to the water (R). Where as a lower score indicates higher relative right frontal activity, thus less reward motivation (NR). Data revealed that FAI decreased by a mean of $0.055 \pm 0.193$ from eu-hydrated to hypohydrated states, with eu-hydration and hypo-hydration FAI of 0.06 and 0.004 respectively. In total, $37.4 \%$ of participants showed an increased affinity(R) for water from eu-hydrated to hypo-hydrated states. Pre and post FS were $2.91 \pm 1.51$ and $.45 \pm 2.88$, respectively. Pre and post FAS were $2.73 \pm .91$ and $2.73 \pm 1.49$. DISCUSSION: Reward/non-reward motivational orientations (affinity to water) changes were less than anticipated following hypo-hydration trials. However, data revealed that $37.4 \%$ of participants exhibited an increased affinity for water according to the FAI scale, supporting the hypothesis. It is possible that $2 \%$ body mass loss is insufficient to create distinct motivational orientation changes within the present sample. Due to the small sample size of the current study, little can be concluded thus far. Additional research addressing the aforementioned methods, as well as larger sample sizes is warranted. If motivational orientation state variability can be established during eu-hydrated and hypo-hydrated states, it may be possible to identify the consumptive tendencies of fluids among individuals. 